\title{
Attractor neural networks with activity-dependent synapses: The role of synaptic facilitation
}

\author{
J.J. Torres ${ }^{\mathrm{a}, *}$, J.M. Cortes ${ }^{\mathrm{b}}$, J. Marro ${ }^{\mathrm{a}}$, H.J. Kappen ${ }^{\mathrm{c}}$ \\ a Department of Electromagnetism and Physics of the Matter, Institute "Carlos I" for Theoretical and Computational Physics University of Granada, \\ E-18071 Granada, Spain \\ ${ }^{\mathrm{b}}$ Institute for Adaptive and Neural Computation, School of Informatics, University of Edinburgh, Edinburgh EH1 2QL, Scotland, UK \\ ${ }^{\mathrm{c}}$ Department of Biophysics, Radboud University of Nijmegen, 6525 EZ Nijmegen, The Netherlands
}

\begin{abstract}
We studied an autoassociative neural network with dynamic synapses which include a facilitating mechanism. We have developed a general mean-field framework to study the relevance of the different parameters defining the dynamics of the synapses and their influence on the collective properties of the network. Depending on these parameters, the network shows different types of behaviour including a retrieval phase, an oscillatory regime, and a non-retrieval phase. In the oscillatory phase, the network activity continously jumps between the stored patterns. Compared with other activity-dependent mechanisms such as synaptic depression, synaptic facilitation enhances the network ability to switch among the stored patterns and, therefore, its adaptation to external stimuli. A detailed analysis of our system reflects an efficient-more rapid and with lesser errors - network access to the stored information with stronger facilitation. We also present a set of Monte Carlo simulations confirming our analytical results.
\end{abstract}

(C) 2006 Elsevier B.V. All rights reserved.

Keywords: Activity-dependent synaptic processes; Attractor neural networks; Switching behaviour

\section{Introduction}

Associative memory illustrates the simplest possible manner in which collective neural computation can work. The basic problem is to store a set of different patterns in a recurrent network, in such a way that when a new stimulus is presented, the network dynamics drives the system to the most similar - according to a previously defined distanceamong all different ones. Thus, the recall of stored patterns during the dynamics appears, and the patterns are the attractors of the dynamics. The number of different attractors determines the number of patterns that the system can store and retrieve. Many different variants, including asymmetric synapses, have been studied in the last decades [2,3]. In these models, after learning, neurons are considered as the only dynamical variables. That is, synapses are static, with fixed strengths. However, it has recently been emphasized that a neural dynamics with

\footnotetext{
*Corresponding author.

E-mail address: jtorres@onsager.ugr.es (J.J. Torres).
}

static synapses is incomplete and unrealistic [1]. The observation of diverse types of synaptic plasticity and ranges of timescales over which they operate suggests that the dynamics of synapses has an active role in information processing in neural systems. Therefore, more realistic neural networks models should include both neural and synaptic dynamical descriptions.

We address here this problem by studying the effect of the consideration of a phenomenological model of dynamic synapses [7] within the context of attractor neural networks (ANN). Although we are concerned here with a network of binary neurons, previous studies have shown that the behaviour of such a simple network dynamics agrees qualitatively with the behaviour that is observed in more realistic networks, such as integrate and fire neuron models of pyramidal cells [5]. Concerning dynamic synapses, there are several activity-dependent processes which take place. For instance, processes in which the postsynaptic potential or response can either decrease (depression) or increase (facilitation) due to repeated presynaptic activation. This has been reported to be 
necessary to produce an appreciable synaptic plasticity [1] which is fundamental for the development and adaptation of the nervous system, and they are believed to be on the basis of higher functions as learning and memory.

\section{The model}

Our starting point is a network of $N$ binary neurons $s_{i}=1,0$ representing, respectively, firing or silent states. Each neuron follows the probabilistic dynamics:

$\operatorname{Prob}\left\{s_{i}(t+1)=1\right\}=\frac{1}{2}\left\{1+\tanh \left[2 \beta h_{i}(t)\right]\right\}$.

$\beta=1 / T$ where $T$ is the temperature, and $h_{i}(t)$ represents the total presynaptic current arriving to the postsynaptic neuron $i$. In order to consider dynamic synapses with facilitation and/or depression, we assume $h_{i}(t)=$ $\sum_{j=1}^{N} \omega_{i j} \mathscr{D}_{j}(t) \mathscr{F}_{j}(t) s_{j}(t)-\theta_{i}$. Here, $\theta_{i}$ is the threshold of neuron $i$ to fire, and $\omega_{i j}$ are the static synaptic weights where the system stored patterns of information during a previous slow learning process. The stored patterns are codified in states of neural activity, namely, $\xi^{v} \equiv\left\{\xi_{i}^{v}\right\}, v=$ $1, \ldots, P$. For instance, one can choose the standard covariance rule, namely, $\omega_{i j}=1 /(N f(1-f)) \sum_{v=1}^{P}\left(\xi_{i}^{v}-\right.$ $f)\left(\xi_{j}^{v}-f\right)$ with $\left\langle\xi_{i}^{v}\right\rangle=f$. The complete dynamics for depression $\mathscr{D}_{j}(t)$ and facilitation $\mathscr{F}_{j}(t)$ was reported in [7]. Here, we use a simplified version of such model in which $\mathscr{D}_{j}(t) \equiv x_{j}(t)$ and $\mathscr{F}_{j}(t) \equiv U+(1-U) u_{j}(t)$, being $x_{j}(t)$ the fraction of neurotransmitters which are in a recovered state. A fraction of these neurotransmitters, namely, $U x(t)$, is ready to be released after the arrival of a presynaptic action potential $\left(s_{j}=1\right)$. The remaining, $(1-U) x(t)$, can also be released by facilitating mechanisms whose dynamics is driven by the variable $u_{j}(t)$. For simplicity we assume that the complete dynamics is described by the discrete system

$$
\begin{aligned}
x_{j}(t+1)= & x_{j}(t)+\frac{1-x_{j}(t)}{\tau_{\mathrm{rec}}}-U x_{j}(t) s_{j}(t) \\
& -(1-U) u_{j}(t) x_{j}(t) s_{j}(t), \\
u_{j}(t+1)= & u_{j}(t)-\frac{u_{j}(t)}{\tau_{\mathrm{fac}}}+U\left[1-u_{j}(t)\right] s_{j}(t),
\end{aligned}
$$

where $\tau_{\text {rec }}$ and $\tau_{\text {fac }}$ are the time constant for depressing and facilitating mechanisms, respectively.

\section{Results}

We solved the system described by Eqs. (1)-(2) within the standard mean field assumption $s_{i} \approx\left\langle s_{i}\right\rangle$ and in the limit of only one stored pattern, $\alpha \equiv P / N=0$ and $f=\frac{1}{2}$, although most of our conclusions are also valid for many patterns. The result is a discrete map $\vec{y}_{t+1}=\vec{F}\left(\vec{y}_{t}\right)$ where $\vec{y} \equiv\left\{m_{+}, m_{-}, x_{+}, x_{-}, u_{+}, u_{-}\right\}$is a vector whose components are order parameters which measure the overlap with the stored pattern $(m)$, the mean depression level $(x)$ and the mean facilitation level $(u)$, in the neurons that are, respectively, active $(+)$ or inactive $(-)$ in the pattern. The local stability of the steady state solutions can be studied by analysing the behavior of the eigenvalues, namely $\lambda_{i}$ associated to the local dynamics of this map (see Ref. [6] for details). In particular, fixed points become unstable when the maximum absolute value of all eigenvalues, namely $|\lambda|_{\max }$ is bigger than one. Fig. 1(left) shows $|\lambda|_{\max }$ as a function of $\tau_{\text {rec }}$ for $U=T=0.1$ and $\tau_{\text {fac }}=20 \mathrm{~ms}$. Then, the analysis of the stability of fixed points reveals three different regimes in the behaviour of the system. First, a ferromagnetic-like phase associated to standard associative memory appears for $\tau_{\text {rec }}<\tau_{\text {rec }}^{*}$. Second a paramagnetic-like or non-memory phase occurs for $\tau_{\text {rec }}>\tau_{\text {rec }}^{* *}$. Finally, an oscillatory phase in which the network activity is jumping
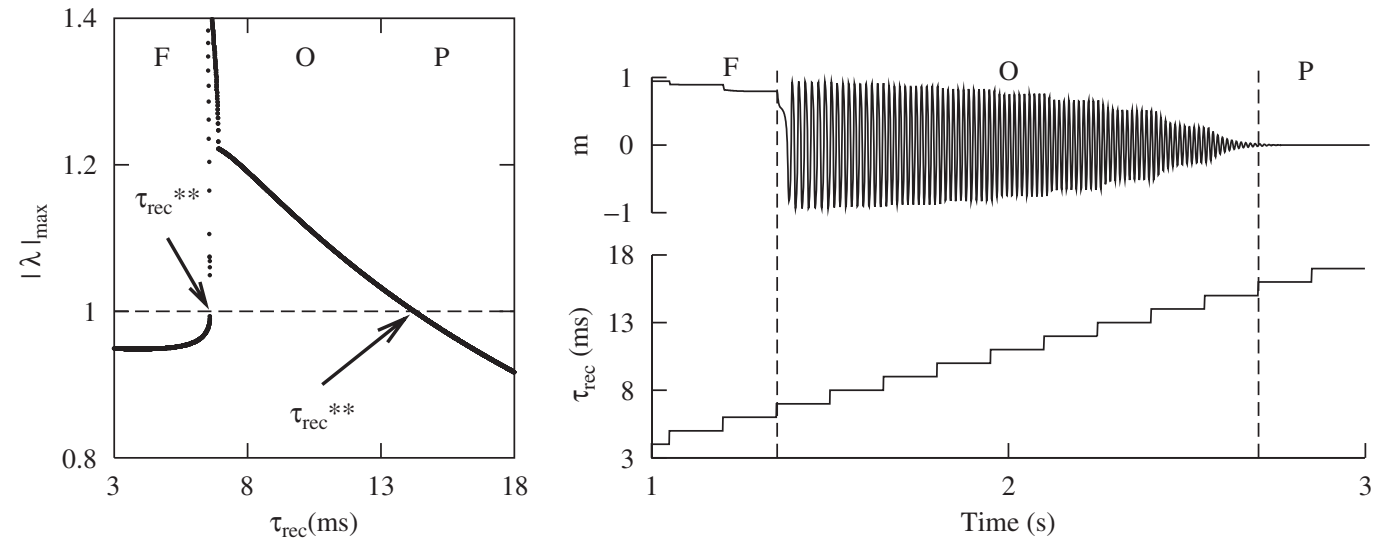

Fig. 1. (Left) Behaviour of the maximum absolute value for the eigenvalues driving the dynamics around the fixed points, for $U=T=0.1$ and $\tau_{\mathrm{fac}}=20 \mathrm{~ms}$. Here, $\tau_{\mathrm{rec}}^{*}$ and $\tau_{\mathrm{rec}}^{* *}$ are, respectively, the critical points at which the ferromagnetic $(\mathrm{F})$ and oscillatory phases $(\mathrm{O})$ become unstable. For $\tau_{\text {rec }}>\tau_{\text {rec }}^{* *}$ the paramagnetic states $(\mathrm{P})$ are the only one that remain stable. (Right) the emergence of different dynamical behaviours by continously increasing $\tau_{\text {rec }}$ from 4 to 18 during $3 \mathrm{~s}$. 

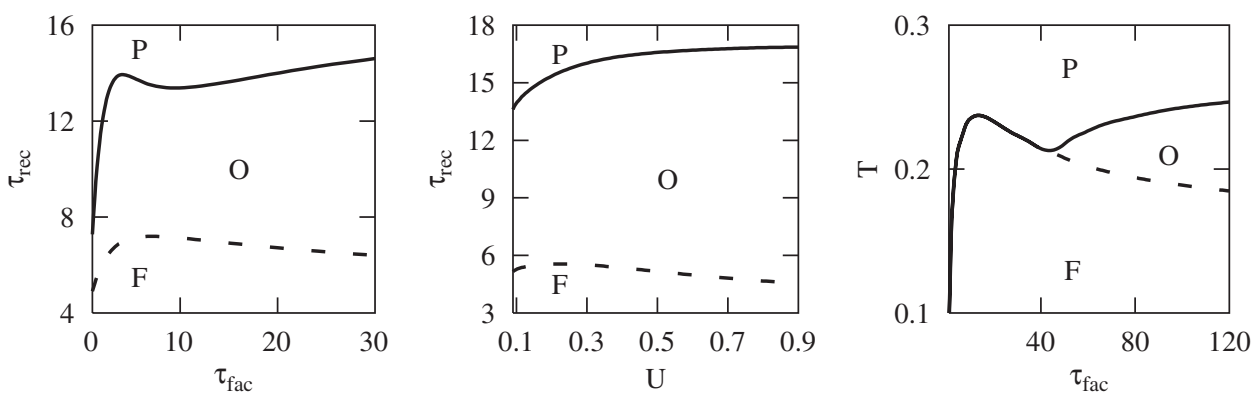

Fig. 2. Phase diagrams for $\alpha=0$, for several values of the relevant parameters defining the dynamic of the synapses, namely $U, \tau_{\text {rec }}, \tau_{\text {fac }}$ and $T$. The left panel represents the phase diagram in the plane $\left(\tau_{\mathrm{rec}}, \tau_{\mathrm{fac}}\right)$ at temperature $T=0.1$ and $U=0.1$. The middle panel corresponds to the phase diagram in the plane $\left(\tau_{\text {rec }}, U\right)$ for $T=0.1$ and $\gamma \equiv \tau_{\text {fac }} / \tau_{\text {rec }}=0.25$. The dashed (solid) line correspond to the line of critical $\tau_{\text {rec }}^{*}\left(\tau_{\text {rec }}^{* *}\right)$ where recall (oscillatory) phase disappears. The right panel is the phase diagram in the plane $\left(T, \tau_{\mathrm{fac}}\right)$ for $U=0.1$ and $\tau_{\text {rec }}=3 \mathrm{~ms}$. In all panels solid lines correspond to second order phase transitions and dashed lines to first-order phase transitions.
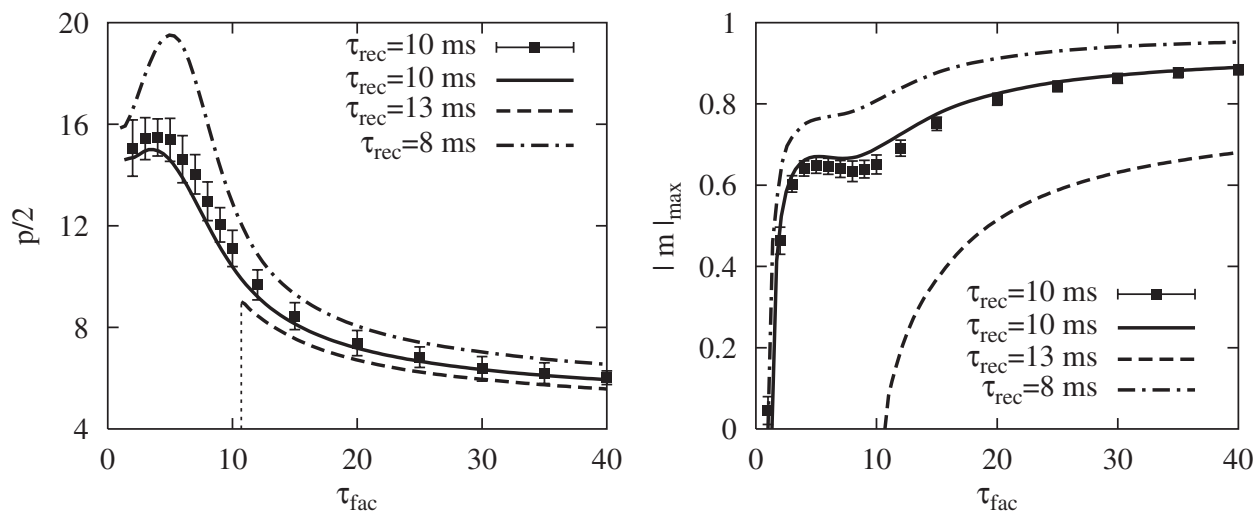

Fig. 3. (Left) Dependence of the half period during the oscillatory regime as a function of $\tau_{\mathrm{fac}}$. (Right) Dependence of the maximum of the overlap $m$ as a function also of $\tau_{\text {fac }}$ and three different values of $\tau_{\text {rec }}$. Both panels show that strong facilitation (large values of $\tau_{\text {fac }}$ ) produces a more rapid access to stored information and with less error. The figure also shows the contrary effect due to depression, that appear for week facilitation (small values of $\tau_{\mathrm{fac}}$ ). Data points correspond to Monte Carlo simulations confirming the mean field results.

between different memories appears for $\tau_{\text {rec }}^{*}<\tau_{\text {rec }}<\tau_{\text {rec }}^{* *}$ Fig. 1(right) shows the emergence of these three phases when one continously varies $\tau_{\text {rec }}$ between 3 and 18 during 3 s. Fig. 2 shows phase diagrams obtained by plotting the critical lines at which transitions between these three phases occur for different values of the parameters $\tau_{\text {rec }}, \tau_{\text {fac }}$ and $U$. By inspection of these diagrams, one observes that the width of the oscillatory phase enlarges for increasing values of $\tau_{\mathrm{fac}}$. Moreover, a detailed analysis of the oscillatory phase shows that the access to the stored memories and the error in the retrieval of such memories strongly depends on facilitation and on its competition with depression. This is shown in Fig. 3 where the half period of the oscillations in the overlap with a pattern, $m \equiv m_{+}-m_{-}$, and its maximum absolute value are represented as function of $\tau_{\text {fac }}$, for different values of $\tau_{\text {rec }}$ and $U=T=0.1$.

\section{Discussion}

Using a realistic model of dynamical synapses which includes short-term facilitation and depression, we show that, depending on the competition between these two mechanisms, different type of behaviour occurs in ANN. For low depression, a ferromagnetic or memory phase occurs. For very large depression or facilitation, a paramagnetic or non-memory phase occurs. For intermediate facilitation and/or depression, an oscillatory phase with the network activity jumping between the attractors shows up. We also observed that a high facilitation enhances the network ability to switch among the stored patterns, as well as its adaptation to external stimuli, as it is reported with more detail in [6]. Other interesting new phenomena are, for instance, that the memory phase disappears earlier for a fixed degree of depression and temperature. Moreover, in the oscillatory phase we observe that its width, in the corresponding phase diagram, increases with facilitation, as shown in Fig. 2. In addition, the frequency of the oscillations also increases with facilitation. As a consequence, we conclude that facilitation allows to recover information with less error but during a shorter period of time. This supports the idea that synaptic facilitation has influence in processes of short-term 
memory. The facility to switch could be interesting to code both spatial and temporal information, and could explain, for instance, the spatio-temporal dynamics in the early olfactory processes which is described in [4].

\section{Acknowledgements}

This work was supported by the MEyC-FEDER project FIS2005-00791 and the Junta de Andalucía project FQM165. JMC also acknowledges financial support from EPSRC-funded COLAMN project Ref. EP/CO 10841/1. We thank Jorge F. Mejías for useful discussions.

\section{References}

[1] L.F. Abbott, W.G. Regehr, Synaptic computation, Nature 431 (2004) 796-803.

[2] D.J. Amit, Modeling Brain Function: The World of Attractor Neural Networks, Cambridge University Press, Cambridge, 1989.

[3] J. Hertz, A. Krogh, R.G. Palmer, Introduction to the Theory of Neural Computation, Addison-Wesley, Reading, MA, 1991.

[4] G. Laurent, M. Stopfer, R.W. Friedrich, M.I. Rabinovich, A. Volkovskii, H.D.I. Abarbanel, Odor encoding as an active, dynamical process: experiments, computation and theory, Ann. Rev. Neurosci. 24 (2001) 263-297.

[5] L. Pantic, J.J. Torres, H.J. Kappen, S.C.A.M. Gielen, Associative memory with dynamic synapses, Neural Comput. 14 (2002) 2903-2923.

[6] J.J. Torres, J.M. Cortes, J. Marro, and H.J. Kappen, Competition between synaptic depression and facilitation in attractor neural networks, Neural Comput., 2006, in press, q-bio.NC/0604019.

[7] M. Tsodyks, K. Pawelzik, H. Markram, Neural networks with dynamic synapses, Neural Comput. 10 (1998) 821-835.

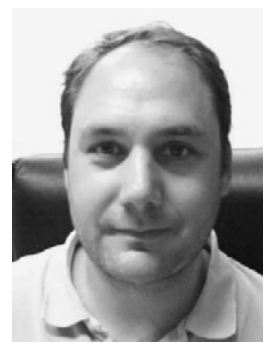

Joaquin J. Torres received his Ph.D. from Granada University in 1997. Between 2001 and 2005 he has been a postdoctoral researcher ("Ramon y Cajal" contract) at Department of Electromagnetism and Physics of the Matter at Granada University. Since 2005, he is an associate professor at the same department and, his current research interests are the mathematical modeling of bio-inspired neural networks and the study of the biophysics of dynamical processes in living cells.

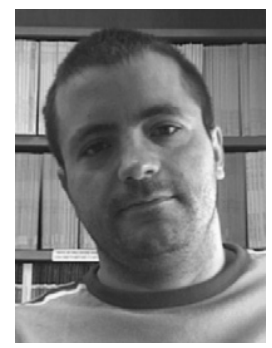

Jesus Cortes earned a Ph.D. in NeuroPhysics at University of Granada, Spain. Because the brain is a complex system par excellence, he is interested how in simple neural systems, emerging collective phenomena appear due to synaptic interaction among neurons. In precisely, he studies how short-term plasticity at synaptic level affects global network behavior in systems neuroscience. Presently, he is a postdoctoral researcher at the Institute for Adaptive and Neural Computation (ANC) at University of Edinburgh, UK.

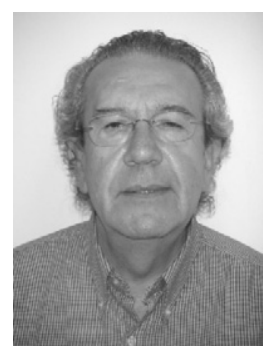

Joaquin Marro earned a Ph.D. in theoretical physics (Barcelona, 1972) and in computational statistical physics (Yeshiva University of New York, 1975). He has contributed on different topics of statistical and condensed matter physics by making a creative use of computers. Marro, who has been visiting several Universities, joined in 1987 the faculty at Granada University where he is presently professor of condensed matter physics and Director of the Department.

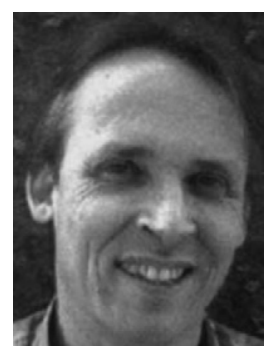

Hilbert J. Kappen studied particle physics in Groningen, The Netherlands, and completed his Ph.D. in this field in 1987 at The Rockefeller University in New York. From 1987 until 1989 he worked as a scientist at the Philips Research Laboratories in Eindhoven, The Netherlands. Since 1989, he is conducting research on neural networks at the laboratory for biophysics of the University of Nijmegen, The Netherlands. Since 1997 he is associate professor and since 2004 full professor at this university. His research is focused on machine learning (stochastic processes, learning algorithms, probabilistic reasoning and several applications in collaboration with industry) and computational neuroscience. He is the director of the Dutch Foundation for Neural Networks (SNN). 